\title{
Electrochemical Functionalization of Graphene at the Nanoscale with Self-Assembling Diazonium Salts
}

\author{
Zhenyuan $\mathrm{Xia}^{1}$, Francesca Leonardi ${ }^{1}$, Marco Gobbi $^{2}$, Yi Liu ${ }^{3}$, Vittorio Bellani ${ }^{4}$, Andrea Liscio ${ }^{1}$, \\ Alessandro Kovtun ${ }^{1}$, Rongjin $\mathrm{Li}^{3}$, Xinliang Feng ${ }^{3}$, Emanuele Orgiu ${ }^{2}$, Paolo Samorì ${ }^{2}$, Emanuele Treossi ${ }^{1^{*}}$, \\ Vincenzo Palermo ${ }^{1 *}$ \\ ${ }^{1}$ Istituto per la Sintesi Organica e la Fotoreattività - Consiglio Nazionale delle Ricerche, via Gobetti 101, \\ 40129 Bologna, Italy \\ ${ }^{2}$ ISIS \& icFRC Université de Strasbourg \& CNRS, 8 Allée Monge, 67000, Strasbourg, France \\ ${ }^{3}$ Max Planck Institute for Polymer Research, Ackermannweg 10, Mainz 55128, Germany \\ ${ }^{4}$ Dipartimento di Fisica, Università degli Studi di Pavia, via Bassi 6, 27100 Pavia, Italy \\ *emanuele.treossi@isof.cnr.it; vincenzo.palermo@isof.cnr.it
}

\begin{abstract}
We describe a fast and versatile method to functionalize high-quality graphene with organic molecules by exploiting the synergistic effect of supramolecular and covalent chemistry. With this goal, we designed and synthesized molecules comprising a long aliphatic chain and an aryl diazonium salt.

Thanks to the long chain these molecules physisorb from solution onto CVD graphene or bulk graphite, self-assembling in an ordered monolayer. The sample is successively transferred into an aqueous electrolyte, to block any reorganization or desorption of the monolayer. An electrochemical impulse is used to transform the diazonium group into a radical capable of grafting covalently to the substrate and transforming the physisorption into a covalent chemisorption. During covalent grafting in water the molecules retain the ordered packing formed upon self-assembly.

Our two-step approach is characterized by the independent control over the processes of immobilization of molecules on the substrate and their covalent tethering, enabling fast $(t<10 \mathrm{sec})$ covalent functionalization of graphene. This strategy is highly versatile and works with many carbon-based materials including graphene deposited on silicon, plastic and quartz, as well as highly oriented pyrolytic graphite.
\end{abstract}

KEYWORDS: graphene, electrochemistry, diazonium salts, self-assembly 
Single layer graphene (SLG), a two-dimensional honeycomb structure of carbon atoms, is a material of great interest because of its remarkable electronic, mechanical, optical and thermal properties. ${ }^{1}$

Nowadays, chemical vapour deposition (CVD) is commonly used to produce meter-size, large area SLG. ${ }^{2}$ The perfect crystal structure of pristine graphene determines its unique properties. However, such an ideal lattice brings about some drawbacks, including its low processability and poor tunability of the electronic properties. As an example, for applications in electronics, graphene-based transistors show charge mobilities much greater than those of silicon, but the absence of a finite band-gap causes a very poor $\mathrm{I}_{\mathrm{on}^{-}}{ }^{-}$ $\mathrm{I}_{\text {off }}$ current ratio. ${ }^{1}$

The creation of chemical defects on the graphene lattice enables an easier processability and more effective tuning of graphene properties. ${ }^{3-4}$ The most common way to functionalize graphene is via vigorous chemical oxidation processes, yielding graphene oxide (GO). Oxygen-functional groups exposed on the basal plane and on the edges of GO create $s p^{3}$ hybridized bonds, thus they disrupt the conjugated $s p^{2}$ network of graphene thereby modifying its unique electrical properties. In such a way, the conductivity of single sheets can be lowered leading to a transition from semi-metal to insulator. ${ }^{5}$

Direct covalent functionalization of pristine graphene (without passing through GO intermediates) can also be obtained with different approaches, taking advantage of the large expertise acquired on the chemistry of carbon nanotubes and fullerenes. ${ }^{6-8}$ However, the low reactivity of the graphene basal surface requires highly reactive conditions for its covalent modification. Although thermal or photoinduced [2+1] cycloadditions of graphene with nitrene and carbene intermediates are commonly employed, the long reaction time and/or low yield hinders an efficient covalent functionalization of graphene. ${ }^{9}$

It was recently demonstrated that graphene functionalization is significantly enhanced when the reaction is carried out under electrochemical control. ${ }^{10-13}$ The electrochemical potential applied can shift the Fermi level of graphene, increasing its reactivity ${ }^{14}$ as compared to direct attack of the covalent $s p^{2}$ bonds with aggressive chemicals.

The resulting structures show a highly disordered morphology on the nanoscale, with a large number of $s p^{3}$ defects that are spatially disordered and poorly controllable. ${ }^{15-17}$ On the other hand, spatially-controlled functionalization of graphene at the nanoscale would allow useful optical and electronic properties to be obtained, such as the creation of a finite bandgap in graphene.

Among the various electrochemical approaches that have been proposed, the electrochemical grafting of diazonium salts ensures a stable grafting of aryl molecules in a short reaction time leading to a high surface coverage..$^{18,19}$ To date, this technique has been used on different kinds of carbon-based surfaces, and it has been demonstrated as a fast and versatile method to graft a wide range of molecules due to the 
easy synthesis of suitable precursors ${ }^{18-19}$. From a mechanistic point of view, the electrochemical reduction of an aryl diazonium salt leads to the formation of a neutral aryl radical which is able to react with carbon-based electrodes (fig. 1a). The use of diazonium salts and electrochemistry allows a facile functionalization (fig. 1b,c); however, this method also features a poor spatial control since it relies on random diffusion of the diazo-radicals from the solution to the target surface. The macroscopic reservoir of molecules in solution provides a practically unlimited amount of active radicals that could further react with the grafted aromatic molecules on graphene, yielding a poly-aryl multilayer of uncontrolled structure and thickness. ${ }^{20} 21$

Here we report an approach to covalently functionalize pristine high-quality SLG obtained by chemical vapour deposition (CVD). Our approach is a two-step process to allow independent control over the adsorption of the molecules on graphene as ruled by supramolecular, non-covalent interactions, and their subsequent covalent grafting to graphene (fig. 1d-f). To this end we have designed and synthesized 4docosyloxy-benzenediazonium tetrafluoroborate (DBT, fig. 2a), an aryl diazonium salt comprising a long aliphatic chain and a diazonium grafting unit. The alkoxy chain promotes the physisorption of DBT on graphene, forming ordered 2D patterns ${ }^{22}$. The highly reactive diazonium salt headgroup allows the covalent attachment of DBT to graphene, disrupting its $s p^{2}$ covalent lattice and thus modifying its optical and electronic properties.

In our approach, DBT is first physisorbed from solution onto graphene, allowing the molecules to selfassemble into ordered monolayers on the surface (fig. 1d). The maximal amount of molecules deposited on the graphene surface depends on the packing density of the DBT monolayer, and can be as low as few nanograms $/ \mathrm{cm}^{2}$. The sample is afterwards transferred in an aqueous electrolyte $\left(0.1 \mathrm{M} \mathrm{H}_{2} \mathrm{SO}_{4}\right)$ that is a non-solvent for DBT. The supramolecular arrangement of the layer is thus fixed, because the molecules cannot desorb or rearrange in such a non-solvent (fig. 1e). Finally, a single electrochemical potential scan triggers the generation of a uniform layer of aryl radicals (fig. 1f), enabling the efficient covalent grafting of graphene in the timescale of a few seconds.

\section{Results and Discussion}

The target diazonium compound DBT was prepared by a three-step route of alkoxy substitution, nitroreduction and diazotisation processes, as portrayed in fig. S1 and described in the Supporting Information. The synthetic steps are all simple and have a high yield, making possible to produce the docosyloxysubstituted aromatic diazonium compound on the gram scale. Because of its long alkoxy chain, the DBT molecule exhibits a high affinity for the graphite surface. ${ }^{22-23}$ Such a high affinity is a key to the formation 
of crystalline physisorbed self-assembled monolayers on graphite. Fig. $2 \mathrm{~b}, \mathrm{c}, \mathrm{d}$ show typical images of the lamellar structure formed on graphite and graphene by DBT as monitored by Scanning Tunneling Microscopy (STM). The quality of the image on single-layer graphene is not as good as on graphite, as a result of the natural roughness of the underlying $\mathrm{SiO}_{\mathrm{x}}$ surface, which has a roughness of few $\AA$, thus causing a blurring effect. However, both data on graphite and graphene show a similar morphology, confirming that the molecules self-assemble into physisorbed monolayers on graphite and graphene in the same way.

In the observed structure the bright part of the image can be ascribed to the conjugated head-group, while the darker part can be attributed to the docosyl chains, which are packed in a side-to-side manner. The 2D pattern is characterized by a unit cell of $\mathrm{a}=(3.9 \pm 0.1) \mathrm{nm}, \mathrm{b}=(1.0 \pm 0.1) \mathrm{nm}$ and $\alpha=\left(89 \pm 2^{\circ}\right)$, with a unit cell area $\mathrm{A}=3.9 \pm 0.2 \mathrm{~nm}^{2}$ containing two molecules per cell, corresponding to a molecules surface density value of $5.3 \times 10^{13} \mathrm{~cm}^{-2}$. The fuzzy appearance of the bright parts where the head-groups are located may be explained by the presence of the $\mathrm{BF}_{4}^{-}$counter-ions weakly interacting with the graphite surface, therefore undergoing dynamics on a timescale faster than the STM imaging. Various experimental proofs support this interpretation. First, there is a wide amount of published data demonstrating that polar moieties and conjugated head groups appear as bright spots when observed in STM experiments (see for instance ref. ${ }^{24}$ and ${ }^{25}$ )

Then, a self-assembled layer composed by just the DBT cations would feature strong electrostatic repulsion due to the close vicinity of positive charges of the $\mathrm{N}_{2}^{+}$groups; therefore the presence of the $\mathrm{BF}_{4}$ anions close to the cationic head group is required to minimize the mutual electrostatic repulsion. The presence of $\mathrm{BF}_{4}$ ions is also suggested by the observed value of the unit cell main axis, $\mathrm{a}=3.9 \pm 0.1 \mathrm{~nm}$. The contour length of the DBT molecule is $3.39 \mathrm{~nm}$ (with the alkyl tail fully stretched), thus could not explain the observed periodicity. However, the size of $\mathrm{BF}_{4}$ anion solvated in acetonitrile was previously estimated by Gogotsi et al. ${ }^{26}$ to be ca. $0.48 \mathrm{~nm}$, which summed to the $3.39 \mathrm{~nm}$ gives a total unit cell of $3.87 \mathrm{~nm}$, in excellent agreement with the lamella width determined experimentally by STM. In addition, the presence of the $\mathrm{BF}_{4}$ anions is further supported by the XPS data that show a clear $F(1 s)$ peak (as detailed further on in the text).

The equal brightness of adjacent bright stripes suggests that the adjacent molecules assemble with a headto-tail intra-lamella packing as depicted in Fig. 2b. Such a motif is further supported by the minimization of the electrostatic repulsion and the maximization of the electrostatic attraction both at the intra- and inter-lamellar level, and by the improved packing density achieved in an intra-lamellar head-to-tail arrangement, in view of the diverse 2D projection of the van der Waals volume of the different moieties, which is greater for the head-group than for the alkyl chains. 


\section{Conventional, direct electro-grafting from solution.}

We first investigated the grafting of DBT diazonium salt using a conventional electrochemical approach where the molecules are dissolved in bulk amounts of electrolyte solution (fig. 1b,c) as already reported previously. ${ }^{21,27}$ A solution of $0.5 \mathrm{mM}$ DBT in acetonitrile (ACN) was used, with $0.1 \mathrm{M}$ tetrabutylammonium hexafluorophosphate $\left(\mathrm{TBAPF}_{6}\right)$ as supporting electrolyte. Graphene supported on different substrates $\left(\mathrm{SiO}_{\mathrm{x}}\right.$ wafer, poly-ethylene-therephtalate (PET) or quartz) was used as the working electrode; Glassy Carbon (GC) and Highly Oriented Pyrolytic Graphite (HOPG) were also used for comparison. Thanks to a homemade setup based on microelectrodes and a PDMS ring, we could perform measurements on a small, fixed area $\left(0.1 \mathrm{~cm}^{2}\right)$ of each sample using small amounts $(10 \mu \mathrm{L})$ of the electrolyte (see fig. 2e and methods section).

Cyclic voltammetry (CV) is a powerful technique to monitor in situ electron transfer processes during the electrochemical reactions. A broad monoeletronic cathodic peak was observed on all samples by scanning the applied potential from $+0.2 \mathrm{~V}$ to $-0.7 \mathrm{~V}\left(v s . \mathrm{Ag} / \mathrm{Ag}^{+}\right.$) at a scan rate of $100 \mathrm{mV} / \mathrm{s}$ (see Fig. S2 in SI). This peak, present only in the first scan and disappearing in the following ones, can be ascribed to the grafting of the DBT to the basal plane of graphite/graphene. This electrochemical evidence was attributed to the reduction of DBT to its radical species (following the reaction scheme in fig. 1a), which immediately reacted with the carbon surface during the first scan. The cathodic grafting of the DBT showed different electrochemical signals depending on the type of working electrodes used, such as CVD-graphene, GC and graphite (as shown in fig. S2a-e). A reference blank solution without DBT molecules was also measured to rule out any influence of the organic electrolyte, showing no reduction peak and a cyclic voltammetry signal stable across several scans (fig. S2f).

After the electrochemical grafting of DBT, the quality of the functionalization was evaluated by using a

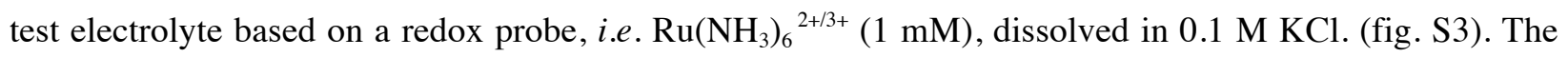
redox peak potential differences $\left(\Delta \mathrm{E}_{\mathrm{p}}\right)$ measured were less than $200 \mathrm{mV}$ before grafting. After grafting, the passivation of the graphene surface due to attachment of the diazonium salt dramatically decreased and broadened the redox signal, and the $\Delta \mathrm{E}_{\mathrm{p}}$ could not be quantified ( $>500 \mathrm{mV}$ ). These results suggest that CVD-graphene, graphite and glassy carbon reacted quickly with DBT in less than 10 seconds using this electrochemical approach. In all cases, the redox probe signal was clearly different on samples grafted with DBT when compared to blank electrodes, with a current decrease of two orders of magnitude. This large decrease of current indicates an effective coating of the conductive areas of the sample with an insulating molecular layer.

The morphology of the graphene after electrografting of DBT was studied by atomic force microscopy (AFM) in intermittent contact mode, showing the typical flat morphology of graphene obtained by CVD 
(fig. S4). The thickness of the pristine and functionalized graphene could be estimated by taking height profiles around the holes in the graphene sheet (which are in practice always present in CVD graphene, due to damage caused during the transfer from copper to the target substrate). Extensive statistical determination of sheet thickness was obtained through the analysis of height histograms ${ }^{28}$ on more than five different holey areas for each sample (total scanned area $\approx 500 \mu \mathrm{m}^{2}$ per sample). Upon DBT grafting, the graphene thickness increased from $1.1 \pm 0.3 \mathrm{~nm}$ to $5.8 \pm 0.2 \mathrm{~nm}$ due to the presence of a uniform, thick organic coating. The increase of thickness was $\approx 4.7 \mathrm{~nm}$, which is much larger than the length of a single layer of DBT, even assuming a fully stretched alkyl chain (3.15 nm after diazonium group removal). The high layer thickness was due to uncontrolled grafting of DBT radicals that react not only with graphene but also with other DBT already grafted, thus forming multilayered branched oligomers on the graphene surface, as observed in previous work. ${ }^{20}$

Raman spectroscopy is able to monitor phonons in graphene, and is thus very sensitive to minimal disruption of the conjugated $s p^{2}$ structure. In particular, after electrografting the presence of defects in the graphene lattice was indicated by the growth of a D peak due to inter-valley resonant Raman scattering (fig. 3a). ${ }^{29}$

The average distance between defective sites $L_{D}$ was calculated from the ratio of the integrated intensity of the $\mathrm{D}$ and $\mathrm{G}$ peaks $I_{D} / I_{G}$, using the formula from ref. ${ }^{30-31}$ :

$$
\frac{I_{D}}{I_{G}}=C_{A} \frac{r_{A}^{2}-r_{S}^{2}}{r_{A}^{2}-2 r_{S}^{2}}\left[\exp \left(-\frac{\pi r_{S}^{2}}{L_{D}^{2}}\right)-\exp \left(-\frac{\pi\left(r_{A}^{2}-r_{S}^{2}\right)}{L_{D}^{2}}\right)\right]+C_{S}\left[1-\exp \left(-\frac{\pi r_{S}^{2}}{L_{D}^{2}}\right)\right]
$$

The details of the calculation are available in the Supporting Information. Raman spectra show that the D peak was negligible in pristine graphene (black line in fig. 3a), confirming the high quality of the starting material and the absence of defects. After electrochemical grafting with DBT there was a large increase in the integrated intensity ratio of the $\mathrm{D}$ and $\mathrm{G}$ peaks $I_{D} / I_{G}$ from 0.28 to 4.0 (table $\mathrm{S} 1$ ), due to the creation of $\mathrm{sp}^{3}$ defects in graphene, with a consequent decrease of $L_{D}$ from $7.4 \mathrm{~nm}$ to $1.4 \mathrm{~nm}$, corresponding to a defect density of $5.3 \times 10^{13} \mathrm{~cm}^{-2}$. These values should be considered indicative, because they depend on the parameters used in eq. 1 , in particular on the assumed radius of the graphene area influenced by each defect (see SI). In any case, the defect density calculated by Raman agrees well with the maximal molecules' density calculated from STM, indicating that the process achieves a dense, although disordered, functionalization of graphene. The ratio between $2 \mathrm{D}$ and $\mathrm{G}$ peak $\left(\mathrm{I}_{2 \mathrm{D}} / \mathrm{I}_{\mathrm{G}}\right)$ also changed upon grafting, going from 3.6 to 1.0 (table S1). This ratio is close to four in good graphene, ${ }^{32}$ but it decreases upon significant doping. 
On the macroscopic scale, significant changes in the electrical resistance of graphene were also measured after grafting. We performed the measurements using two- and four-probe setup. Statistics based on 27 measurements using a two probe system (fig. 4) showed a ten-fold increase in the resistance of graphene electrodes upon electrografting (from $340 \pm 30 \Omega$ to $3600 \pm 400 \Omega$ ). Measurements of sheet resistance using a four probe van der Pauw geometry showed an increase from $600 \pm 90 \Omega /$ sq to $3000 \pm 300$ $\Omega /$ sq after electrografting treatment.

These experiments demonstrated that upon the application of electrochemical potential as low as $-0.2 \mathrm{~V}$ $\left(v s . \mathrm{Ag} / \mathrm{Ag}^{+}\right)$the DBT molecules freely dispersed in bulk amounts of solution could be transformed into reactive radicals and then attached covalently to the graphene surface in a fast and irreversible way. However, the quality of the organic layer in terms of thickness and ordering was not easy to control. Thick layers with no visible ordering were obtained, because the reactive radicals could also attack previously grafted molecules, yielding irregular structures. ${ }^{20}$

\section{Self-assembly molecules prior to the electro-grafting}

As displayed in fig. 1d-f, a more controlled approach to achieve ordered molecular grafting would be to graft pre-deposited, self-assembled monolayers of molecules on the graphene surface. With this aim, we first tested the self-assembling behaviour of DBT deposited from three different organic solvents: chloroform $\left(\mathrm{CHCl}_{3}\right)$, tetrahydrofuran (THF) and acetonitrile $(\mathrm{ACN})$ with a DBT concentration of 0.25 $\mathrm{mg} / \mathrm{mL}(\approx 0.5 \mathrm{mM})$. Each solution was spin-coated onto freshly cleaved graphite surfaces $\left(1.2 \times 1.2 \mathrm{~cm}^{2}\right)$ and studied by AFM (fig. S5). DBT physisorbed on graphite using all three solvents, forming different assemblies. The samples obtained from THF solutions gave irregular amorphous aggregates with lateral size of $\approx 50 \mathrm{~nm}$ and thickness of $\approx 3 \mathrm{~nm}$; conversely, samples prepared using $\mathrm{CHCl}_{3}$ and $\mathrm{ACN}$ as solvents exhibited a layered structure with different morphologies, whose thickness was variable but typically below $2 \mathrm{~nm}$.

In particular AFM images on graphite of DBT deposited from ACN (fig. S6) showed the formation of a physisorbed self-assembled monolayer (SAM), composed of stripes of aligned DBT molecules featuring a periodic motif typical of long alkoxy chains, similar to the case observed by STM on graphene and graphite (fig. 2b-d) but with a larger spacing (see SI for a detailed discussion). A similar morphology was also obtained by spin coating from chloroform, although slightly less uniform.

After having confirmed the successful formation of DBT ordered monolayers on the graphite surface by self-assembly, we performed the electrochemical covalent functionalization of graphene. Herein, in contrast to previous works on diazonium salts functionalization, ${ }^{19-20,33}$ we could use aqueous electrolytes 
(0.1 $\left.\mathrm{M} \mathrm{H}_{2} \mathrm{SO}_{4}\right)$ instead of organic solvents; since DBT is not soluble in water, the ordered SAM was immobilized on the substrate, with no chance of molecular detachment from the surface into the water.

The grafting process was done on graphene monolayers deposited on different substrates, and took place regardless of the nature of the underlying substrate. Fig. 5 displays the CV curve of graphene samples transferred on $\mathrm{SiO}_{\mathrm{x}}$, PET or quartz, then coated with DBT and treated electrochemically by ramping the voltage from $+0.2 \mathrm{~V}$ to $-0.7 \mathrm{~V}$ at $100 \mathrm{mV} / \mathrm{s}$. The broad irreversible peak observed during the first cyclic voltammetry scan is consistent with the reduction of DBT to its radical cations, which rapidly reacted with the graphene surface. This cathodic reduction peak disappeared during the second and third scans. A similar behaviour was also observed for DBT deposited on graphite from all the different solvents used (fig. S7).

Redox reactivity measurements using a $\mathrm{Ru}\left(\mathrm{NH}_{3}\right)_{6}{ }^{2+/ 3+}$ probe showed the formation of an insulating layer on the surface of all samples tested, as shown for graphene (fig. 6) and graphite (fig. S8), in analogy with the results obtained by conventional grafting in solution.

According to the mechanism shown in fig. 1a, successful grafting implies that the DBT molecule loses its charge, and thus its attraction for the $\mathrm{BF}_{4}^{-}$counter-ion. This was confirmed by X-ray photoemission spectroscopy (XPS): fluorine peaks 1s and KLL were observed for the spin-coated DBT layer (fig. 7) indicating the presence of $\mathrm{BF}_{4}^{-}$, since the molecules were still present on graphene in the form of charged salts. Conversely, after grafting and washing steps, the fluorine peaks disappeared, indicating that the ordered structures observed by AFM were composed of neutral molecules.

The ordered structure, composed of parallel stripes, survived the electrochemical treatment, and the lamellar packing could still be observed, at least on graphite, by STM (fig. S9) and by AFM (fig. S10, see additional text in SI for more details); STM and AFM measurements were not successful instead on the graphene surfaces, due to the atomic level substrate roughness previously mentioned and to the graphene substrate being much less conductive after grafting (see below).

It should be noted that, in absence of covalent grafting, the removal of $\mathrm{BF}_{4}^{-}$counter-ions (shown by XPS) would destabilize the ordered packing leading to desorption of the molecules or at least a strong rearrangement of the layer morphology. The fact that the lamellar packing is still present after grafting and washing (see SI) indicates that the molecules are not charged anymore, but have been covalently grafted to the surface following the scheme in fig. 1a.

Raman characterization of graphene after DBT self-assembly+grafting is shown in fig. $3 b$ (to be compared with fig. 3a). Table S1 compares the evolution of different Raman observables of graphene after either direct grafting form solution or self-assembly+grafting.

The $I_{D} / I_{G}$ ratio increased from 0.28 to 0.94 , indicating a the creation of $s p^{3}$ defects, but in a much lower amount than in the case of direct grafting from solution $\left(I_{D} / I_{G}=4.0\right)$. No significant change $\mathrm{I}_{2 \mathrm{D}} / \mathrm{I}_{\mathrm{G}}$ was 
instead observed after self-assembly+grafting, again indicating a significantly lower amount of defects created on graphene as compared to direct grafting from solution.

The average distance between reacted sites observed with self-assembly+grafting (calculated with eq. 1) was $L_{D} \approx 3.8 \mathrm{~nm}$, which is comparable to the molecular packing as measured by STM, corresponding to a density of $6.7 \times 10^{12} \mathrm{~cm}^{-2}$, significantly lower than what was obtained by direct functionalization from solution. Electrical characterization measured using a van der Pauw geometry indicated that the sheet resistance value of the modified graphene surface was significantly higher $(2000 \pm 200 \Omega / \square)$ than the untreated one, although the increase was lower than in the case of direct grafting from solution.

The chemically modified sample shows an average thickness of $2.0 \pm 0.3 \mathrm{~nm}$ including the SLG layer (fig. S11), measured by extensive AFM topographical profiles taken on holey parts of the SLG film. This thickness is much smaller than the $5.8 \mathrm{~nm}$ obtained with conventional electrochemical grafting (fig. S4). After subtracting the thickness of uncoated graphene $(1.1 \pm 0.3 \mathrm{~nm})$ it gives a thickness of the grafted DBT layer of $\approx 0.9 \mathrm{~nm}$, thus confirming that the two-step approach can avoid the formation of a multi-layered aryl-functionalized film and that the molecules lay relatively flat on the substrate. Combining the DBT surface density (as measured by STM) and the DBT molecular weight of $401 \mathrm{~g} / \mathrm{mol}$ (with no diazonium group), a DBT coating of $\approx 35$ nanograms $/ \mathrm{cm}^{2}$ could be estimated for a uniform, perfect coverage, although the defects observed in the grafted layer will significantly modify this theoretical value.

Changes in electrical properties were measured at the nanoscale using Kelvin Probe Force Microscopy (KPFM, Fig. S12). ${ }^{34}$ This scanning probe, contactless technique, uses the electrostatic interactions of a metallic AFM tip to measure quantitatively the electric surface potential $(S P)$ of a substrate.

Pristine graphene samples on SiOx treated by electrochemistry without any DBT molecules gave $\mathrm{SP}=$ $230 \pm 10 \mathrm{mV}$, comparable to those measured on pristine graphene ( $\mathrm{SP}=245 \pm 10 \mathrm{mV})$. This confirmed that the solvents used and the electrochemical treatment did not significantly modify graphene, although minimal changes due to doping impurities could always be present. Conversely, graphene coated with DBT molecules gave a significantly lower potential amounting to $130 \pm 10 \mathrm{mV}$, likely due to the presence of $\mathrm{BF}_{4}^{-}$anions. After self-assembly+grafting, the surface potential value increased to $305 \pm 10 \mathrm{mV}$. The positive change of surface potential $(\Delta=60 \pm 14 \mathrm{mV})$ after functionalization indicates that $p$-alkoxyphenyl groups works as electron donors and permanently lift the potential value of graphene, which is in good agreement with previous results. ${ }^{35}$

In summary, the results obtained with CV, STM, AFM, XPS, KPFM and conductivity measurements at the macroscale indicate that the properties of graphene were significantly modified by the deposition and 
electrochemical grafting, and that DBT molecules could be grafted on all the surfaces studied: bulk graphite, glassy carbon and graphene on three different substrates $\left(\mathrm{SiO}_{\mathrm{x}}, \mathrm{PET}\right.$ and quartz).

\section{Conclusions}

The two-step electrochemical reduction process that we used allows good control over the morphology of the grafted layer, because the DBT molecules did not react randomly on the substrate from the bulk electrolyte, but were pre-assembled on the substrate using a suitable solvent. After self-assembly, the DBT layer could be efficiently grafted onto graphene by applying a small negative potential $(-0.2 \mathrm{~V} v s$. $\mathrm{Ag} / \mathrm{Ag}^{+}$). Successful grafting of the graphene surface was demonstrated by significant changes in the electrochemical activity of the substrate, while at the same time the patterned nano-structure of the organic layer was maintained.

Diazonium chemistry has already been used to functionalize graphene. However, a major shortcoming of diazonium chemistry is the limited covalent grafting density in combination with multilayer formation or dendritic growth. ${ }^{20}$ Application of electrochemical bias can greatly enhance grafting yield by tuning the Fermi level of graphene, thereby increasing the rate of the electron transfer reaction. The negative potential applied to graphene also concentrates the diazonium cations within the double layer to accelerate the reaction, but the position of the grafted molecules is always random. ${ }^{33}$ Dendritic growth can be hindered by functionalizing the grafting molecules with bulky side groups, ${ }^{20}$ but this limits the number of molecules that can be used, and requires additional synthetic steps.

Our approach builds on these previous works by combining self-assembly of long alkyl chains to preposition the molecules before fixing them. Noteworthy, Figure 13 of ref. ${ }^{20}$ shows long alkyl chains and diazonium salts as separate molecules on graphite. Instead, we combined the covalent grafting unit and the self-assembling moieties in a single molecule to exploit this spontaneous self-assembly to form ordered, periodically-spaced covalent grafting of graphene and graphite.

This procedure may be compared to previously published approaches that used ordered layers of nanoparticles $^{36-37}$ or block copolymers ${ }^{38-40}$ as nano-masks for selective cutting or patterning of graphene; however, using such masks, patterns with a periodicity $>10 \mathrm{~nm}$ were typically obtained. Conversely, the use of self-assembling molecules leads to periodicities well below $10 \mathrm{~nm}$, dictated only by the length and self-assembling behaviour of the molecules used. Both properties may be tuned very well using synthetic chemistry. Supramolecular self-assembly of organic molecules on graphite is a well-known technique, studied since the invention of STM, and it allows to control the morphology of the self-assembled layers obtaining highly ordered arrays because of the reversible nature of the supramolecular interactions involved. . $^{23,41-42}$ 
The electrochemical approach used here to functionalize graphene is fast and simple because it does not require additional reactants and can be performed in a few seconds using a simple setup. We demonstrated the versatility of this approach by using it on different carbon-based materials, utilizing a simple homemade electrochemical setup. Besides the easy packing of the aliphatic chains used here, the two-step approach can also be used for more complex 2D patterns, e.g. based on arrays formed by adsorption of alternating complementary building blocks or 2-dimensional nanoporous organic frameworks, paving the way to a versatile, well-controlled and simple route to functionalize such a technologically important yet poorly reactive material.

\section{METHODS}

\section{CVD-graphene preparation and transfer process}

The single-layer graphene (SLG) samples were grown on $\mathrm{Cu}$ foil through a chemical vapour deposition (CVD) process. Then, SLG was transferred onto different substrates using a polymer-supported wet transfer method: a PMMA solution (Poly(methyl methacrylate), 4\% in anisole, MICRO CHEM) was spin-coated on CVD graphene/copper foils at $2000 \mathrm{rpm}$ for 1 minute and dried in air at $100{ }^{\circ} \mathrm{C}$. Iron (III) Chloride (Sigma Aldrich) was used to etch the copper for 5 hours, and then the PMMA/graphene film was rinsed in deionized (DI) water several times to remove the etchant residue. The thin film was further cleaned by floating on $10 \% \mathrm{HCl}$ solution and repeated rinsing in DI water several times. Then the film was fished from the water surface and deposited on three different substrates: silicon coated with a 300 $\mathrm{nm}$ thick thermally grown $\mathrm{SiO}_{\mathrm{x}}$ layer, poly-ethylene-terephthalate (PET) or quartz. After drying for 12 hours at room temperature, the substrate was baked at $160{ }^{\circ} \mathrm{C}$ in air for $30 \mathrm{~min}$. Then, the PMMA supporting layer was removed with an acetone bath for 1 hour.

\section{Electrochemical grafting of aryl diazonium salt}

The synthetic route of the 4-docosyloxy-benzenediazonium tetrafluoroborate (DBT) molecule is portrayed in fig. S1. Full characterization of the different intermediates by nuclear magnetic resonance (NMR) is reported in fig. S14-S19.

A DBT solution $(0.25 \mathrm{mg} / \mathrm{mL}, \sim 0.5 \mathrm{mM})$ in chloroform, acetonitrile (ACN) or tetrahydrofuran (THF) was spin-coated $(2000 \mathrm{rpm}, 1 \mathrm{~min})$ onto CVD-graphene $\left(1 \times 1 \mathrm{~cm}^{2}\right)$ supported on either $\mathrm{SiO}_{x}$, quartz or PET substrates.

Dipping the sample in a solution is an easy way to coat a surface; however, the final result depends on how the macroscopic layer of solution remaining on the surface evaporates, leading in some cases to the occurrence of irregular and uncontrolled dewetting (see as example ${ }^{43}$ ). Conversely, spin-coating is a very 
efficient technique for deposition of molecules on surfaces, conventionally used as example to obtain uniform layers of resist in the microelectronics industry. In spin-coating, due to the centrifugal force applied to the spinning sample, most of the deposited solution is spun out of the sample surface leaving only a thin coating layer, which evaporates in a uniform way. Overall, dipping gives the molecules more time to self-assemble in ordered layers, but is less uniform on a large scale; spin-coating can give less ordered systems, but it is more reproducible on large scale.

After drying the sample in air at RT, a polydimethylsiloxane (PDMS) circular mask was fixed on the top of graphene sample along with an electrochemical setup composed of a three-electrode cell: a Pt wire as counter electrode, $\mathrm{Ag} / \mathrm{Ag}^{+}$as reference electrode and the target substrate (graphene etc.) as working electrode (fig. 2e). The PDMS mask confined laterally the electrolytic solution giving a fixed reaction area of $\approx 0.1 \mathrm{~cm}^{2}$ on the CVD graphene, also making it possible to perform electrochemical treatments with very small amounts $(10 \mu \mathrm{L})$ of solution.

Then, an acidic solution $\left(0.1 \mathrm{M} \mathrm{H}_{2} \mathrm{SO}_{4}\right)$ was deposited inside the mask as the electrolyte. The DBT molecules were not soluble in this acidic aqueous solution. After electrochemical reaction, graphene samples were sequentially washed by DI water and dried under a gentle flow of nitrogen gas.

Highly Oriented Pyrolytic Graphite (HOPG) electrodes were also treated in the same way for comparison. The two-step treatment was compared also with a more conventional, direct electrochemical treatment in a solution of DBT salt $(0.5 \mathrm{mM})$ and tetrabutylammonium hexafluorophosphate $\operatorname{TBAPF}_{6}(0.1 \mathrm{M})$ in an organic electrolyte $(\mathrm{ACN})$. In the electrografting procedure, all potentials were quoted with respect to a $\mathrm{Ag} / \mathrm{Ag}^{+}$reference electrode, constructed by placing a silver wire in a solution of $0.01 \mathrm{M} \mathrm{AgNO}_{3}$ and 0.1

$\mathrm{M} \mathrm{TBAPF}_{6}$ in $\mathrm{ACN}$ overnight. After all electrochemical treatments, graphene samples were repeatedly washed by acetonitrile and chloroform to remove the diazonium salt residue.

We cannot exclude some degree of spontaneous reduction of the DBT molecule in acetonitrile. However, the DBT molecule under study was stable in the solvent used, and no significant differences in the grafting process were observed upon using freshly prepared solutions or solutions stored for one day in dark. It should be noted that a previous work ${ }^{44}$ studied the deposition on graphene of a similar molecule, 4-bromobenzenediazonium tetrafluoroborate (4-BBD, $1 \mathrm{mM}$ in a 1:1 mixture of water and methanol for 2 h). Only a slight change in the Raman $\mathrm{D}$ peak and a decrease in $2 \mathrm{D} / \mathrm{G}$ intensity were observed indicating that, in absence of any electrochemical treatment, 4-BBD was a stable dopant on graphene via physisorption.

\section{Electrochemical characterization}


The electrochemical characterization was performed using a circular PDMS mask in a three-electrode configuration cell connected to a potentiostat $\mu$-Autolab type III (Metrohm Italia s.r.l., Varese, Italy). Graphene, Pt and Ag acted as working (WE), counter (CE) and reference (RE) electrodes, respectively. $\mathrm{CV}$ measurements were performed by following the redox signal of $\mathrm{Ru}\left(\mathrm{NH}_{3}\right)_{6}{ }^{2+/ 3+}(1 \mathrm{mM})$ with $0.1 \mathrm{M}$ $\mathrm{KCl}$ as supporting electrolyte. Electrochemical impedance spectroscopy analysis (EIS) measurements, reported in SI, were recorded in a frequency range of $100 \mathrm{kHz}-0.1 \mathrm{~Hz}$ with an AC perturbation of $5 \mathrm{mV}$.

\section{Structural, spectroscopic and electrical characterization}

Scanning Tunneling Microscopy (STM) measurements were carried out by using a Veeco Scanning Tunneling Microscope (multimode Nanoscope III, Veeco) operating with a piezoelectric scanner which allowed the mapping of a maximum area of $1 \times 1 \mu \mathrm{m}^{2}$. As substrates, we used highly oriented pyrolytic graphite (HOPG) and commercial CVD graphene supported on $\mathrm{Si}_{/} \mathrm{SiO}_{2}(300 \mathrm{~nm}$ thick). The graphene sample was used as received, without any additional cleaning step, and was stored in air for two months before the STM experiments. The substrates were glued onto a magnetic disk and an electric contact was made with silver paint (Aldrich Chemicals).

The STM tips were mechanically cut from a Pt/Ir wire (90/10, diameter $0.25 \mathrm{~mm}) .80 \mu \mathrm{l}$ of a $160 \mu \mathrm{g} / \mathrm{ml}$ concentrated solution of DBT were spin coated on the freshly-cleaved HOPG substrate or on the CVD graphene to obtain the self-assembled films. To minimize the effect of water condensing in the tip-tosurface junction, the STM measurements were performed by immersing the tip inside a drop of highly apolar non-solvent, i.e. 1-phenyloctane, which was drop-cast on the pre-assembled organic film prior to the measurement. Alternatively, images were also recorded in air, although at lower resolution. The raw STM data were processed through the application of background flattening. In the case of the HOPG image in fig. $2 \mathrm{~b}$, the drift of the piezo was corrected by using the underlying hexagonal graphite lattice as a reference. The lattice of the underlying substrate was visualized by lowering the bias voltage $\left(\mathrm{V}_{\mathrm{t}}\right)$ to $10 \mathrm{mV}$ and keeping the same average current $\mathrm{I}_{\mathrm{t}}=60 \mathrm{pA}$. In STM imaging both current and height signals were recorded.

AFM imaging was carried out with a Digital Instruments AFM (NT-MDT) operating in intermittent contact mode. Nanoprobe cantilevers (Model: RTESP, Material: 1-10 Ohm-cm Phosphorus (n) doped Si, $\mathrm{f}_{0}=27-309 \mathrm{kHz}, \mathrm{k}=20-80 \mathrm{~N} \cdot \mathrm{m}^{-1}$; from Bruker) were used. KPFM measurements were obtained in air by employing a commercial microscope Multimode (Bruker) with Extender Electronics module using Pt/Ir coated ultra-lever silicon tips ( $\mathrm{SCM}$, Bruker, $\mathrm{k}=2.8 \mathrm{~N} \cdot \mathrm{m}^{-1}$ ) with oscillating frequencies in the range between 60-90 KHz. 
The resistance of the patterned graphene electrodes was measured by a homemade two/four-probe system (Keithley 6517A/Keithley 6514). CVD-graphene samples $\left(1 \times 20 \mathrm{~mm}^{2}\right)$ on $\mathrm{Si} / \mathrm{SiO}_{\mathrm{x}}$ substrates were vertically patterned with $\mathrm{Ag}$ channels $\left(1 \times 10 \mathrm{~mm}^{2}\right.$, thickness $\left.75 \mathrm{~nm}\right)$ by thermal evaporation, creating conductive channels of size $1 \times 1 \mathrm{~mm}^{2}$. A four-probe setup was used to rule out any effect of contact resistance. X-ray photoemission spectroscopy (XPS) was performed exploiting an ultrahigh vacuum (UHV) apparatus (base pressure $1 \times 10^{-10}$ mbar) using a non-monochromatic $\mathrm{Mg} \mathrm{K \alpha}$ excitation source (XR-50, Specs) and a hemispherical energy analyser (Phoibos 100, Specs). Only XPS of fluorine could be measured, while XPS of carbon and oxygen did not give significant results due to the presence of solvents and residues from CVD transfer. Raman spectroscopy measurements were carried out with a microRaman spectrometer (LabRAM by Horiba Jobin-Yvon), using a 100× objective (laser spot diameter $\approx 2$ $\mu \mathrm{m})$, laser excitation wavelength of $632.8 \mathrm{~nm}$ and laser power $\approx 4 \mathrm{~mW} .{ }^{1} \mathrm{H}$ NMR and ${ }^{13} \mathrm{C}$ NMR spectra were recorded in deuterated solvents on a Bruker DPX 300. To avoid artifacts due to variation of Raman signal across the sample, the reported values are averages of Raman measurements performed on different points of the samples' surface. The D, G and 2D Raman peaks have been best-fitted using Lorentzian line-shapes to obtain the reported values of the Raman parameters.

Unless otherwise noted, all starting materials and solvents were purchased from Aldrich and Acros, and used as received without further purification.

\section{Acknowledgments}

We acknowledge the European Union Seventh Framework Programme under grant agreement nº69656 Graphene Flagship, the EC Marie-Curie ITN-iSwitch (GA no. 642196) and the FET project UPGRADE (project no. 309056). We also acknowledge the Agence Nationale de la Recherche through the LabEx

project Chemistry of Complex Systems (ANR-10-LABX-0026_CSC), and the International Center for Frontier Research in Chemistry (icFRC).

\section{Supporting Information}

The supporting Information is available free of charge on the ACS Publications website at DOI: . Synthesis of aryl diazonium salt, Raman analysis, AFM measurements on graphite before and after grafting, extra CV, AFM, STM and EIS results.

Corresponding Authors

E-mail: emanuele.treossi@isof.cnr.it 
E-mail: vincenzo.palermo@isof.cnr.it

Notes

The authors declare no competing financial interest. 


\section{References}

1. $\quad$ Ferrari, A. C.; Bonaccorso, F.; Fal'ko, V.; Novoselov, K. S.; Roche, S.; Boggild, P.; Borini, S.; Koppens, F. H. L.; Palermo, V.; Pugno, N., et al., Science and Technology Roadmap for Graphene, Related Two-Dimensional Crystals, and Hybrid Systems. Nanoscale 2015, 7, 4598-4810.

2. $\quad$ Lin, Y.-C.; Jin, C.; Lee, J.-C.; Jen, S.-F.; Suenaga, K.; Chiu, P.-W., Clean Transfer of Graphene for Isolation and Suspension. Acs Nano 2011, 5, 2362-2368.

3. Borini, S.; White, R.; Wei, D.; Astley, M.; Haque, S.; Spigone, E.; Harris, N.; Kivioja, J.; Ryhanen, T., Ultrafast Graphene Oxide Humidity Sensors. Acs Nano 2013, 7, 11166-11173.

4. Prezioso, S.; Perrozzi, F.; Giancaterini, L.; Cantalini, C.; Treossi, E.; Palermo, V.; Nardone, M.; Santucci, S.; Ottaviano, L., Graphene Oxide as a Practical Solution to High Sensitivity Gas Sensing. $J$. Phys. Chem. C 2013, 117, 10683-10690.

5. Vianelli, A.; Candini, A.; Treossi, E.; Palermo, V.; Affronte, M., Observation of Different Charge Transport Regimes and Large Magnetoresistance in Graphene Oxide Layers. Carbon 2015, 89, 188-196.

6. Quintana, M.; Vazquez, E.; Prato, M., Organic Functionalization of Graphene in Dispersions. Acc. Chem. Res. 2013, 46, 138-148.

7. $\quad$ Englert, J. M.; Dotzer, C.; Yang, G. A.; Schmid, M.; Papp, C.; Gottfried, J. M.; Steinruck, H. P.; Spiecker, E.; Hauke, F.; Hirsch, A., Covalent Bulk Functionalization of Graphene. Nature Chemistry 2011, 3, 279-286.

8. Hirsch, A.; Englert, J. M.; Hauke, F., Wet Chemical Functionalization of Graphene. Acc. Chem. Res. 2013, 46, 87-96.

9. Lawrence, E. J.; Wildgoose, G. G.; Aldous, L.; Wu, Y. A.; Warner, J. H.; Compton, R. G.; McNaughter, P. D., 3-Aryl-3-(Trifluoromethyl)Diazirines as Versatile Photoactivated "Linker" Molecules for the Improved Covalent Modification of Graphitic and Carbon Nanotube Surfaces. Chem. Mater. 2011, 23, 3740-3751.

10. Xia, Z. Y.; Giambastiani, G.; Christodoulou, C.; Nardi, M. V.; Koch, N.; Treossi, E.; Bellani, V.; Pezzini, S.; Corticelli, F.; Morandi, V., et al., Synergic Exfoliation of Graphene with Organic Molecules and Inorganic Ions for the Electrochemical Production of Flexible Electrodes. ChemPlusChem 2014, 79, 439-446.

11. Cooper, A. J.; Wilson, N. R.; Kinloch, I. A.; Dryfe, R. A. W., Single Stage Electrochemical Exfoliation Method for the Production of Few-Layer Graphene Via Intercalation of Tetraalkylammonium Cations. Carbon 2014, 66, 340-350.

12. Rapino, S.; Treossi, E.; Palermo, V.; Marcaccio, M.; Paolucci, F.; Zerbetto, F., Playing Peekaboo with Graphene Oxide: A Scanning Electrochemical Microscopy Investigation. Chem. Commun. 2014, 50, 13117-13120.

13. Sarkar, S.; Bekyarova, E.; Haddon, R. C., Reversible Grafting of a-Naphthylmethyl Radicals to Epitaxial Graphene. Angew. Chem. Int. Ed. 2012, 51, 4901-4904.

14. Shih, C.-J.; Wang, Q. H.; Jin, Z.; Paulus, G. L. C.; Blankschtein, D.; Jarillo-Herrero, P.; Strano, M. S., Disorder Imposed Limits of Mono- and Bilayer Graphene Electronic Modification Using Covalent Chemistry. Nano Lett. 2013, 13, 809-817.

15. Bagri, A.; Mattevi, C.; Acik, M.; Chabal, Y. J.; Chhowalla, M.; Shenoy, V. B., Structural Evolution During the Reduction of Chemically Derived Graphene Oxide. Nature Chemistry 2010, 2, 581587.

16. Perrozzi, F.; Prezioso, S.; Donarelli, M.; Bisti, F.; De Marco, P.; Santucci, S.; Nardone, M.; Treossi, E.; Palermo, V.; Ottaviano, L., Use of Optical Contrast to Estimate the Degree of Reduction of Graphene Oxide. J. Phys. Chem. C 2013, 117, 620-625.

17. Erickson, K.; Erni, R.; Lee, Z.; Alem, N.; Gannett, W.; Zettl, A., Determination of the Local Chemical Structure of Graphene Oxide and Reduced Graphene Oxide. Adv. Mater. 2010, 22, 4467-4472. 
18. Allongue, P.; Delamar, M.; Desbat, B.; Fagebaume, O.; Hitmi, R.; Pinson, J.; Saveant, J. M., Covalent Modification of Carbon Surfaces by Aryl Radicals Generated from the Electrochemical Reduction of Diazonium Salts. J. Am. Chem. Soc. 1997, 119, 201-207.

19. Gan, L.; Zhang, D.; Guo, X., Electrochemistry: An Efficient Way to Chemically Modify Individual Monolayers of Graphene. Small 2012, 8, 1326-1330.

20. Greenwood, J.; Phan, T. H.; Fujita, Y.; Li, Z.; Ivasenko, O.; Vanderlinden, W.; Van Gorp, H.; Frederickx, W.; Lu, G.; Tahara, K., et al., Covalent Modification of Graphene and Graphite Using Diazonium Chemistry: Tunable Grafting and Nanomanipulation. ACS Nano 2015, 9, 5520-5535.

21. Kariuki, J. K.; McDermott, M. T., Nucleation and Growth of Functionalized Aryl Films on Graphite Electrodes. Langmuir 1999, 15, 6534-6540.

22. Ciesielski, A.; El Garah, M.; Haar, S.; Kovaricek, P.; Lehn, J. M.; Samori, P., Dynamic Covalent Chemistry of Bisimines at the Solid/Liquid Interface Monitored by Scanning Tunnelling Microscopy. Nature Chemistry 2014, 6, 1017-1023.

23. Rabe, J. P.; Buchholz, S., Commensurability and Mobility in 2-Dimensional Molecular-Patterns on Graphite. Science 1991, 253, 424-427.

24. De Feyter, S.; De Schryver, F. C., Two-Dimensional Supramolecular Self-Assembly Probed by Scanning Tunneling Microscopy. Chem. Soc. Rev. 2003, 32, 139-150.

25. Haar, S.; Ciesielski, A.; Clough, J.; Yang, H.; Mazzaro, R.; Richard, F.; Conti, S.; Merstorf, N.; Cecchini, M.; Morandi, V., et al., A Supramolecular Strategy to Leverage the Liquid-Phase Exfoliation of Graphene in the Presence of Surfactants: Unraveling the Role of the Length of Fatty Acids. Small 2015, $11,1691-1702$.

26. Chmiola, J.; Largeot, C.; Taberna, P.-L.; Simon, P.; Gogotsi, Y., Desolvation of Ions in Subnanometer Pores and Its Effect on Capacitance and Double-Layer Theory. Angew. Chem. Int. Ed. 2008, 47, 3392-3395.

27. Lillethorup, M.; Kongsfelt, M.; Ceccato, M.; Jensen, B. B. E.; Jorgensen, B.; Pedersen, S. U.; Daasbjerg, K., High- Versus Low- Quality Graphene: A Mechanistic Investigation of Electrografted Diazonium- Based Films for Growth of Polymer Brushes. Small 2014, 10, 922-934.

28. Liscio, A., Scanning Probe Microscopy Beyond Imaging: A General Tool for Quantitative Analysis. ChemPhysChem 2013, 14, 1283-1292.

29. Ferrari, A. C.; Meyer, J. C.; Scardaci, V.; Casiraghi, C.; Lazzeri, M.; Mauri, F.; Piscanec, S.; Jiang, D.; Novoselov, K. S.; Roth, S., et al., Raman Spectrum of Graphene and Graphene Layers. Phys. Rev. Lett. 2006, 97, \#187401.

30. Lucchese, M. M.; Stavale, F.; Ferreira, E. H. M.; Vilani, C.; Moutinho, M. V. O.; Capaz, R. B.; Achete, C. A.; Jorio, A., Quantifying Ion-Induced Defects and Raman Relaxation Length in Graphene. Carbon 2010, 48, 1592-1597.

31. Wang, Q. H.; Jin, Z.; Kim, K. K.; Hilmer, A. J.; Paulus, G. L. C.; Shih, C.-J.; Ham, M.-H.; Sanchez-Yamagishi, J. D.; Watanabe, K.; Taniguchi, T., et al., Understanding and Controlling the Substrate Effect on Graphene Electron-Transfer Chemistry Via Reactivity Imprint Lithography. Nature Chemistry 2012, 4, 724-732.

32. Casiraghi, C., Raman Spectroscopy of Graphene. In Spectrosc. Prop. Inorg. Organomet. Compd., RSC: 2012; Vol. 43, p 29.

33. Shih, C. J.; Lin, S. C.; Sharma, R.; Strano, M. S.; Blankschtein, D., Understanding the PhDependent Behavior of Graphene Oxide Aqueous Solutions: A Comparative Experimental and Molecular Dynamics Simulation Study. Langmuir 2012, 28, 235-241.

34. Palermo, V.; Morelli, S.; Palma, M.; Simpson, C.; Nolde, F.; Herrmann, A.; Mullen, K.; Samori, P., Nanoscale Structural and Electronic Properties of Ultrathin Blends of Two Polyaromatic Molecules: A Kelvin Probe Force Microscopy Investigation. Chemphyschem 2006, 7, 847-853.

35. Koehler, F. M.; Luechinger, N. A.; Ziegler, D.; Athanassiou, E. K.; Grass, R. N.; Rossi, A.; Hierold, C.; Stemmer, A.; Stark, W. J., Permanent Pattern-Resolved Adjustment of the Surface Potential of Graphene-Like Carbon through Chemical Functionalization. Angew. Chem. Int. Ed. 2009, 48, 224-227. 
36. Liu, L.; Zhang, Y. L.; Wang, W. L.; Gu, C. Z.; Bai, X. D.; Wang, E. G., Nanosphere Lithography for the Fabrication of Ultranarrow Graphene Nanoribbons and on- Chip Bandgap Tuning of Graphene. Adv. Mater. 2011, 23, 1246.

37. Solis-Fernandez, P.; Yoshida, K.; Ogawa, Y.; Tsuji, M.; Ago, H., Dense Arrays of Highly Aligned Graphene Nanoribbons Produced by Substrate-Controlled Metal-Assisted Etching of Graphene. Adv. Mater. 2013, 25, 6562-6568.

38. Liu, G.; Wu, Y.; Lin, Y.-M.; Farmer, D. B.; Ott, J. A.; Bruley, J.; Grill, A.; Avouris, P.; Pfeiffer, D.; Balandin, A. A., et al., Epitaxial Graphene Nanoribbon Array Fabrication Using Bcp-Assisted Nanolithography. ACS Nano 2012, 6, 6786-6792.

39. Kim, J. Y.; Kim, B. H.; Hwang, J. O.; Jeong, S.-J.; Shin, D. O.; Mun, J. H.; Choi, Y. J.; Jin, H. M.; Kim, S. O., Flexible and Transferrable Self-Assembled Nanopatterning on Chemically Modified Graphene. Adv. Mater. 2013, 25, 1331-1335.

40. Kim, S.-S.; Choi, J.-Y.; Kim, K.; Sohn, B.-H., Large Area Tunable Arrays of Graphene Nanodots Fabricated Using Diblock Copolymer Micelles. Nanotechnology 2012, 23,\#125301.

41. Wang, Q. H.; Hersam, M. C., Room-Temperature Molecular-Resolution Characterization of SelfAssembled Organic Monolayers on Epitaxial Graphene. Nature Chemistry 2009, 1, 206-211.

42. Cai, J.; Ruffieux, P.; Jaafar, R.; Bieri, M.; Braun, T.; Blankenburg, S.; Muoth, M.; Seitsonen, A. P.; Saleh, M.; Feng, X., et al., Atomically Precise Bottom-up Fabrication of Graphene Nanoribbons. Nature 2010, 466, 470-473.

43. Palermo, V.; Palma, M.; Tomovic, Z.; Watson, M. D.; Mullen, K.; Samori, P., Self-Assembly of Pi-Conjugated Discs on Heterogeneous Surfaces: Effect of the Micro- and Nano-Scale Dewetting. Synth. Met. 2004, 147, 117-121.

44. $\quad$ Farmer, D. B.; Golizadeh-Mojarad, R.; Perebeinos, V.; Lin, Y.-M.; Tulevski, G. S.; Tsang, J. C.; Avouris, P., Chemical Doping and Electron-Hole Conduction Asymmetry in Graphene Devices. Nano Lett. 2009, 9, 388-392. 


\section{FIGURES CAPTION}

Figure 1. a) Scheme of the electrochemical reduction of diazonium salts and their covalent attachment on the basal plane of graphene. b,c) Scheme of conventional electrografting of molecules on graphene using macroscopic amounts of solution. $\mathrm{d}$,e,f) Scheme of the two-steps self-assembly + electrochemical grafting procedure used in this work.

Figure 2. a) Molecular structure of DBT. b) STM image of DBT assembly on graphite, showing also the unit cell; the possible molecular packing is also schematized. The position of $\mathrm{BF}_{4}^{-}$counterions is just indicative. Lattice parameters: $\left.a=3.9 \pm 0.1 \mathrm{~nm}: \mathrm{b}=1.0 \pm 0.1 \mathrm{~nm}: \mathrm{a}=89^{\circ} \pm 2^{\circ}: A=3.9 \pm 0.1 \mathrm{~nm}^{2} . \mathrm{c}, \mathrm{d}\right) \mathrm{STM}$ height (c) and current (d) images of DBT self-assembled on graphene. The quality of the image on single-layer graphene is not as good as on graphite, as a result of the natural roughness of the underlying $\mathrm{SiO}_{\mathrm{x}}$ surface, which has a roughness of few $\AA$, thus causing a blurring effect. The images were recorded by using the following tunnelling parameters: (b) tip voltage $\left(\mathrm{V}_{\mathrm{t}}\right)=-1200 \mathrm{mV}$ and average tunnelling current $\left(\mathrm{I}_{\mathrm{t}}\right)=60$ $\mathrm{pA}$; (c) and (d) $\left(\mathrm{V}_{\mathrm{t}}\right)=-1200 \mathrm{mV},\left(\mathrm{I}_{\mathrm{t}}\right)=20 \mathrm{pA}$. e) Schematic representation of the electrochemical setup used for grafting. WE (working electrode)= graphene; CE (counter electrode) $\mathrm{Pt}$; RE (reference electrode $)=\mathrm{Ag} / \mathrm{Ag}^{+}$.

Figure 3. a) Raman spectra of CVD-graphene on $\mathrm{SiOx}$ substrates before and after direct electro-grafting of DBT from solution (0.5 mM in ACN). b) Raman spectra of graphene/SiOx substrate after spin coating of DBT $\left(0.5 \mathrm{mM}\right.$ in $\left.\mathrm{CHCl}_{3}\right)$, electrochemical grafting and washing.

Figure 4. a) Statistics of resistance changes in CVD-graphene electrodes reacted with bulk amounts of DBT in ACN solution.

Figure 5. a) b) Cyclic voltammograms of monolayers of DBT deposited from $\mathrm{CHCl}_{3}$ on CVD-graphene, then transferred in 0.1 $\mathrm{M} \mathrm{H}_{2} \mathrm{SO}_{4}$ water solution. Graphene on different substrates ( $\mathrm{SiOx}$, PET or Quartz) is used.

Figure 6. Redox activity of DBT-CVD-graphene on different substrates, in $0.1 \mathrm{M} \mathrm{KCl}$ solution (Redox Probe: $\left.1 \mathrm{mM} \mathrm{Ru}\left(\mathrm{NH}_{3}\right)_{6}{ }^{2+/ 3+}\right)$ before and after grafting of DBT.

Figure 7. XPS spectra of graphene/SiOx substrate before and after spin coating of DBT, electrochemical grafting and washing. 
a)

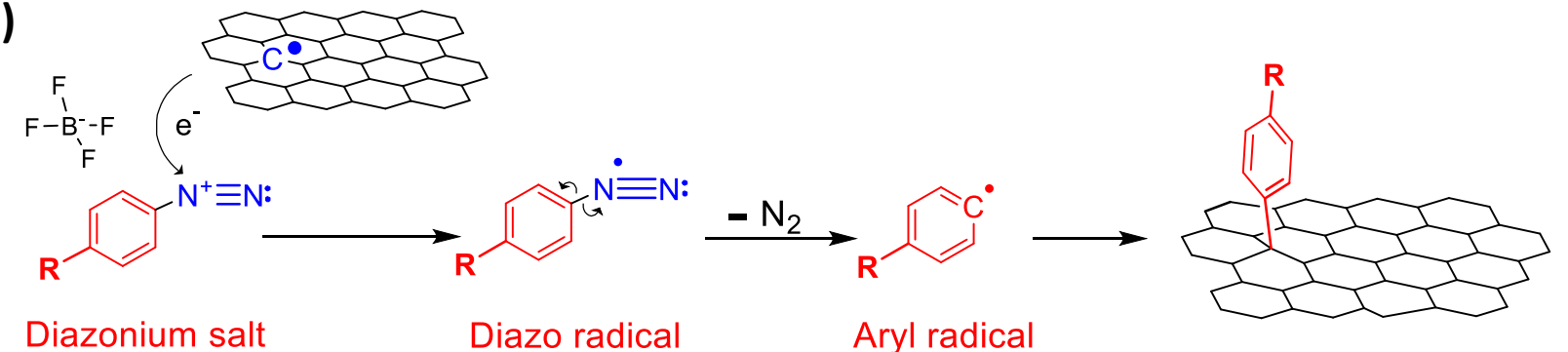

B) MOLECULES IN ORGANIC SOLVENT

C) CONVENTIONAL ELECTROGRAFTING IN SOLUTION
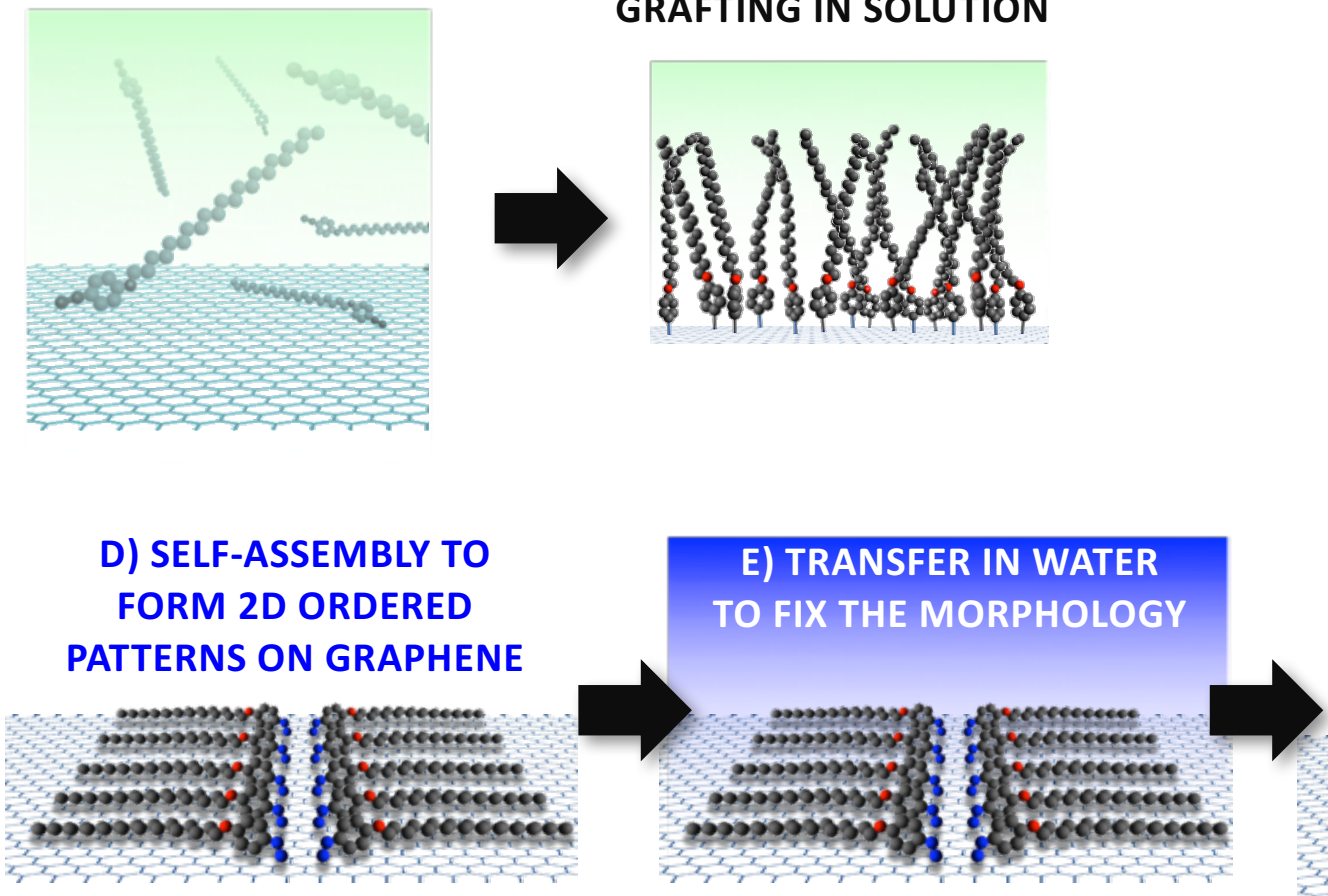

F) COVALENT GRAFTING

OF THE PATTERNED

MOLECULES

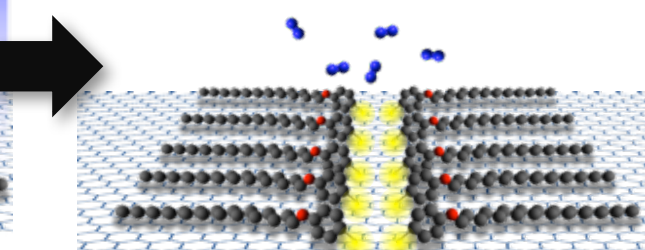

Figure 1. a) Scheme of the electrochemical reduction of diazonium salts and their covalent attachment on the basal plane of graphene. b,c) Scheme of conventional electrografting of molecules on graphene using macroscopic amounts of solution. $d, e, f)$ Scheme of the two-steps selfassembly + electrochemical grafting procedure used in this work. 
a)

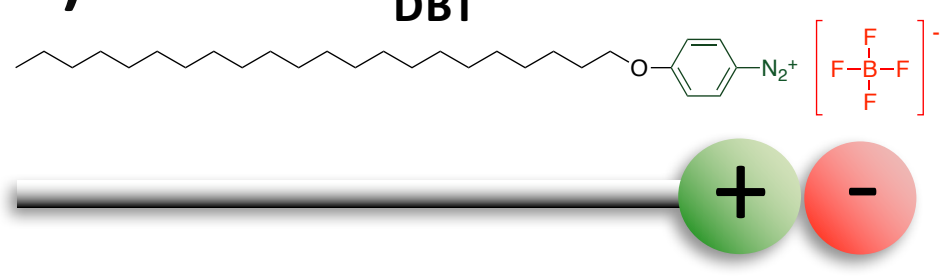

d)

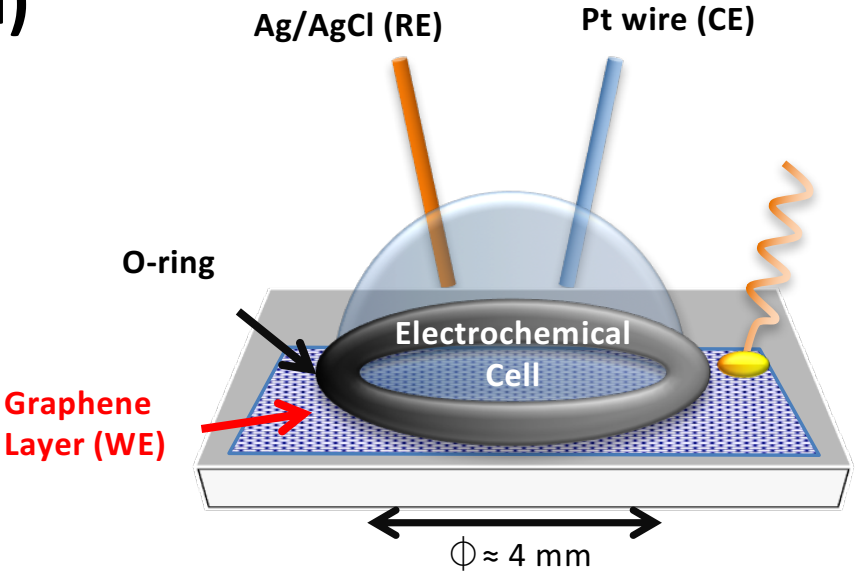

Self-assembly on GRAPHITE

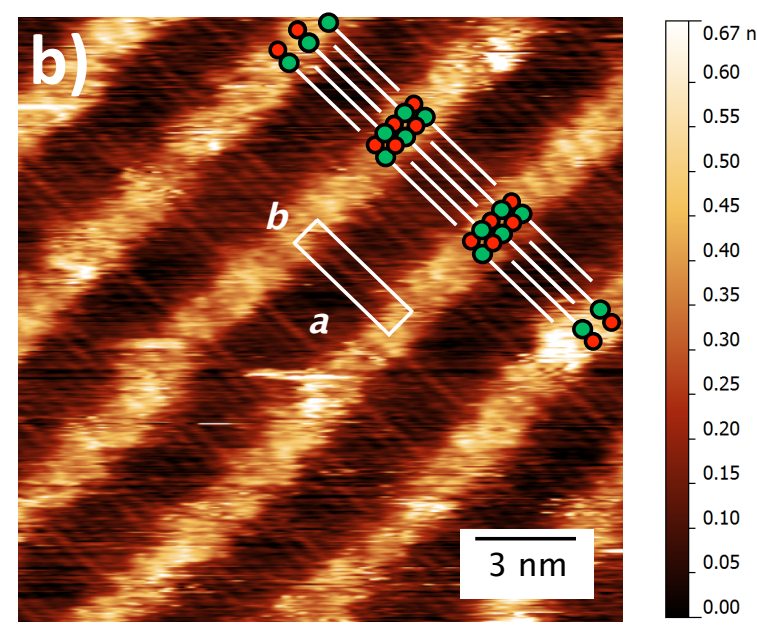

Self-assembly on GRAPHENE

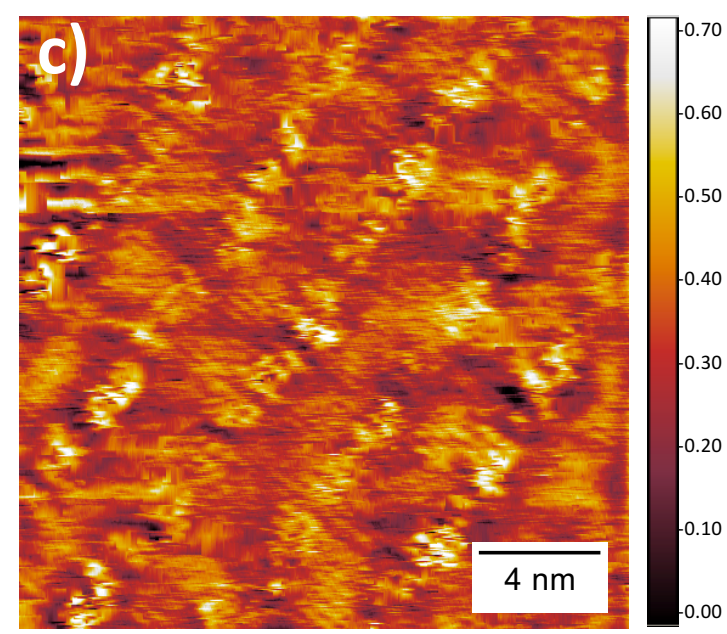

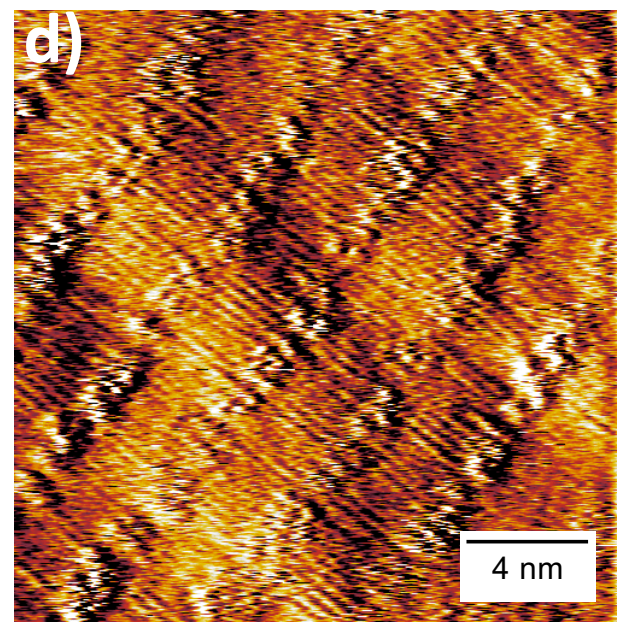

Figure 2. a) Molecular structure of DBT. b) STM image of DBT assembly on graphite, showing also the unit cell; the possible molecular packing is also schematized. The position of $\mathrm{BF}_{4}{ }^{-}$counterions is just indicative. Lattice parameters: $a=3.9 \pm 0.1 \mathrm{~nm}: b=1.0 \pm 0.1 \mathrm{~nm}: a=89^{\circ} \pm 2^{\circ}: A=3.9 \pm 0.1 \mathrm{~nm}{ }^{2}$. c,d) STM height (c) and current (d) images of DBT self-assembled on graphene. The quality of the image on single-layer graphene is not as good as on graphite, as a result of the natural roughness of the underlying SiO $_{x}$ surface, which has a roughness of few $\AA$, thus causing a blurring effect. e) Schematic representation of the electrochemical setup used for grafting. WE (working electrode)= graphene; $C E$ (counter electrode)= Pt; RE (reference electrode) $=\mathrm{Ag} / \mathrm{AgCl}(3 \mathrm{M} \mathrm{KCl})$. 

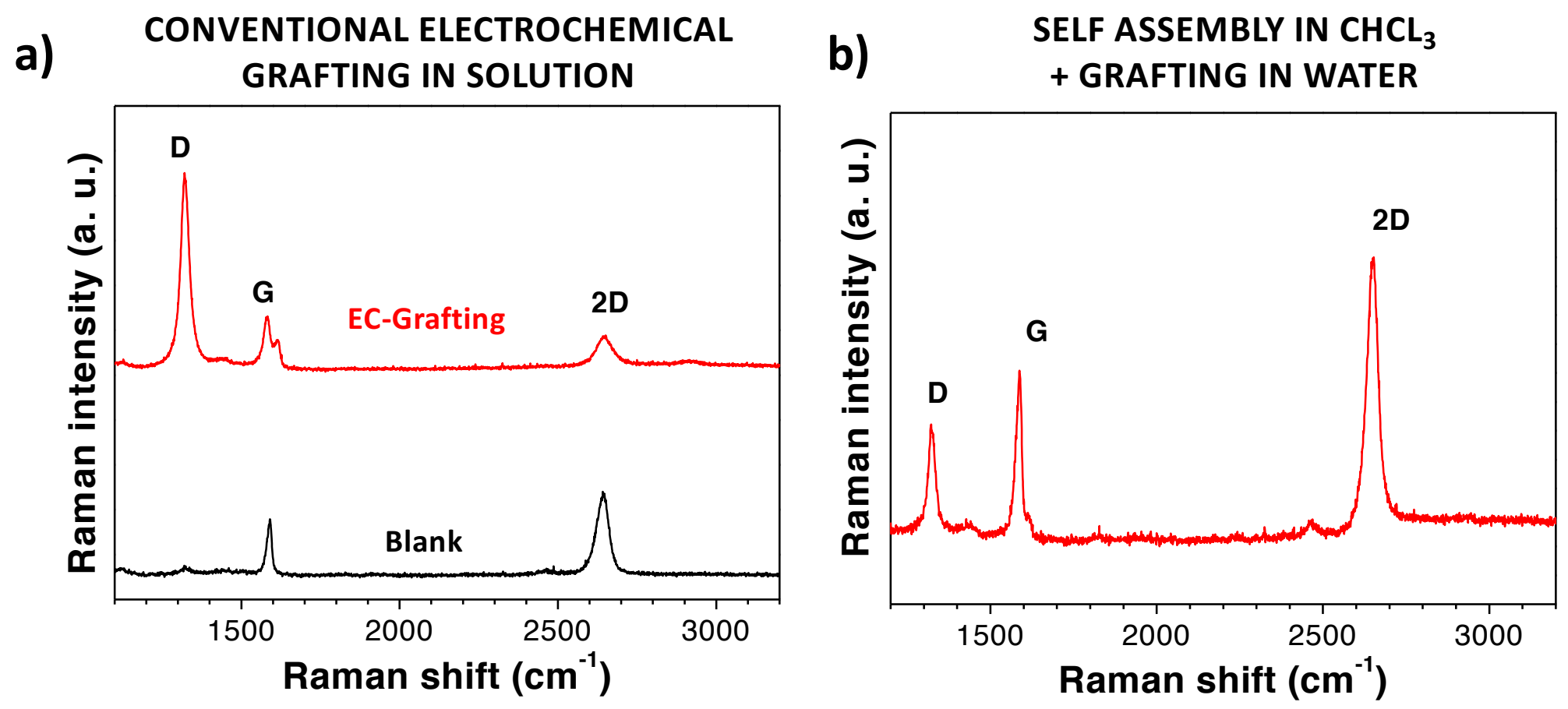

Figure 3. a) Raman spectra of CVD-graphene on SiOx substrates before and after direct electro-grafting of DBT from solution (0.5 mM in ACN). b) Raman spectra of graphene/SiOx substrate after spin coating of DBT, electrochemical grafting and washing. 


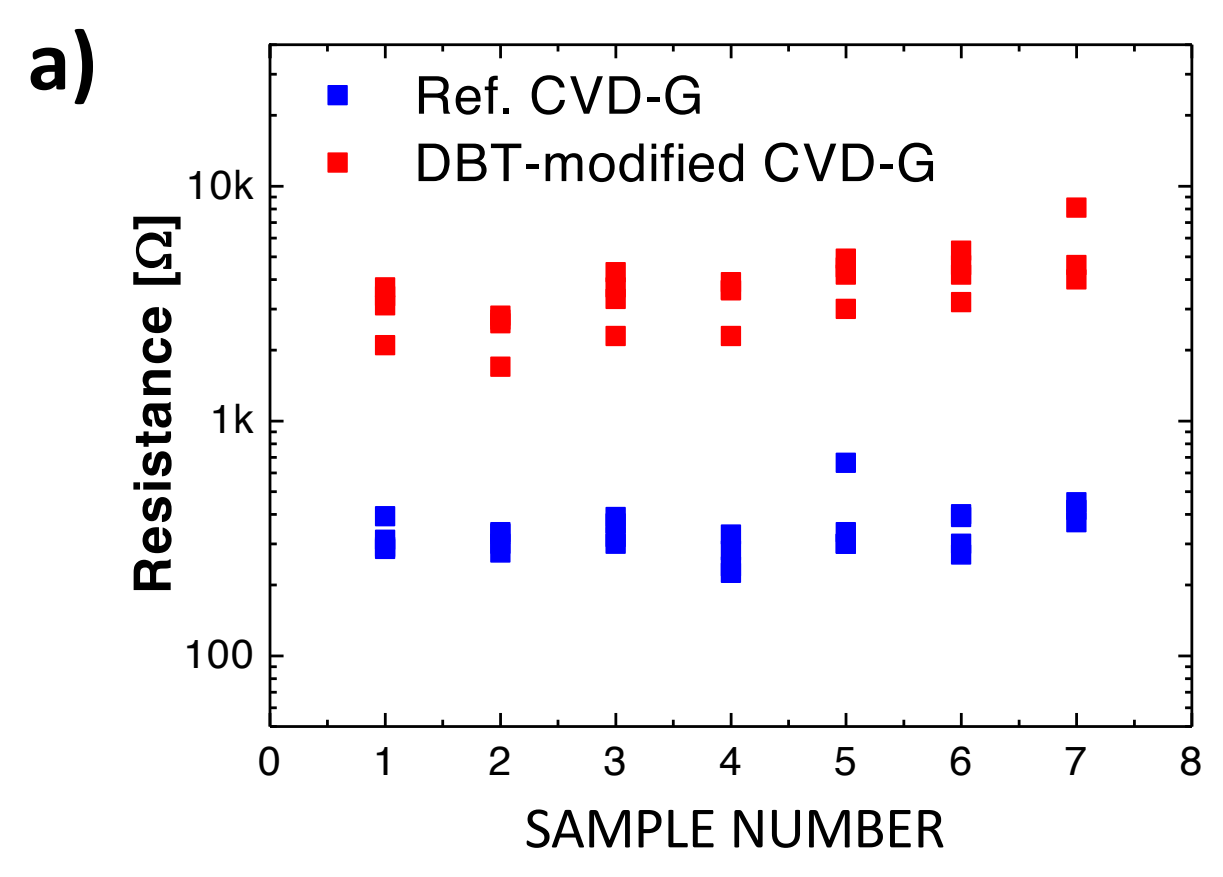

Figure 4. a) Statistics of resistance changes after CVD-graphene reacted with bulk amounts of DBT in ACN solution. 


\section{Self Assembly in $\mathrm{CHCl}_{3}+$ Grafting in Water}
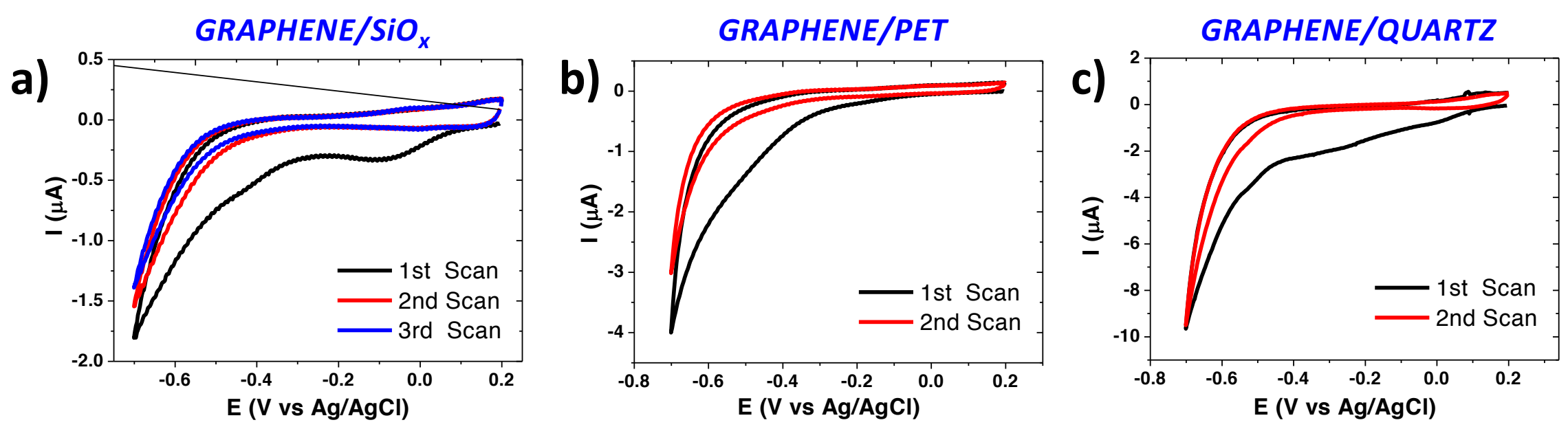

Figure 5. a) b) Cyclic voltammograms of monolayers of DBT deposited from $\mathrm{CHCl}_{3}$ on CVD-graphene, then transferred in $0.1 \mathrm{M} \mathrm{H}_{2} \mathrm{SO}_{4}$ water solution. Graphene on different substrates (SiOx, PET or Quartz) is used. 

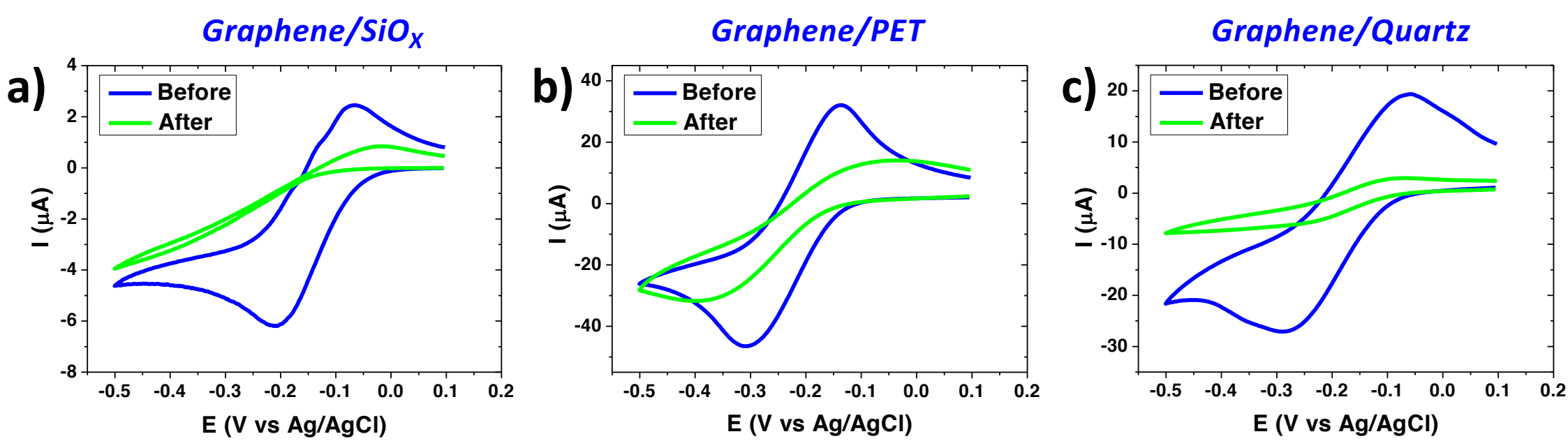

Figure 6. Redox activity of DBT-CVD-graphene on different substrates, in $0.1 \mathrm{M} \mathrm{KCl}$ solution (Redox Probe: $1 \mathrm{mM}$ $\left.\mathrm{Ru}\left(\mathrm{NH}_{3}\right)_{6}{ }^{2+/ 3+}\right)$ before and after grafting of DBT. 


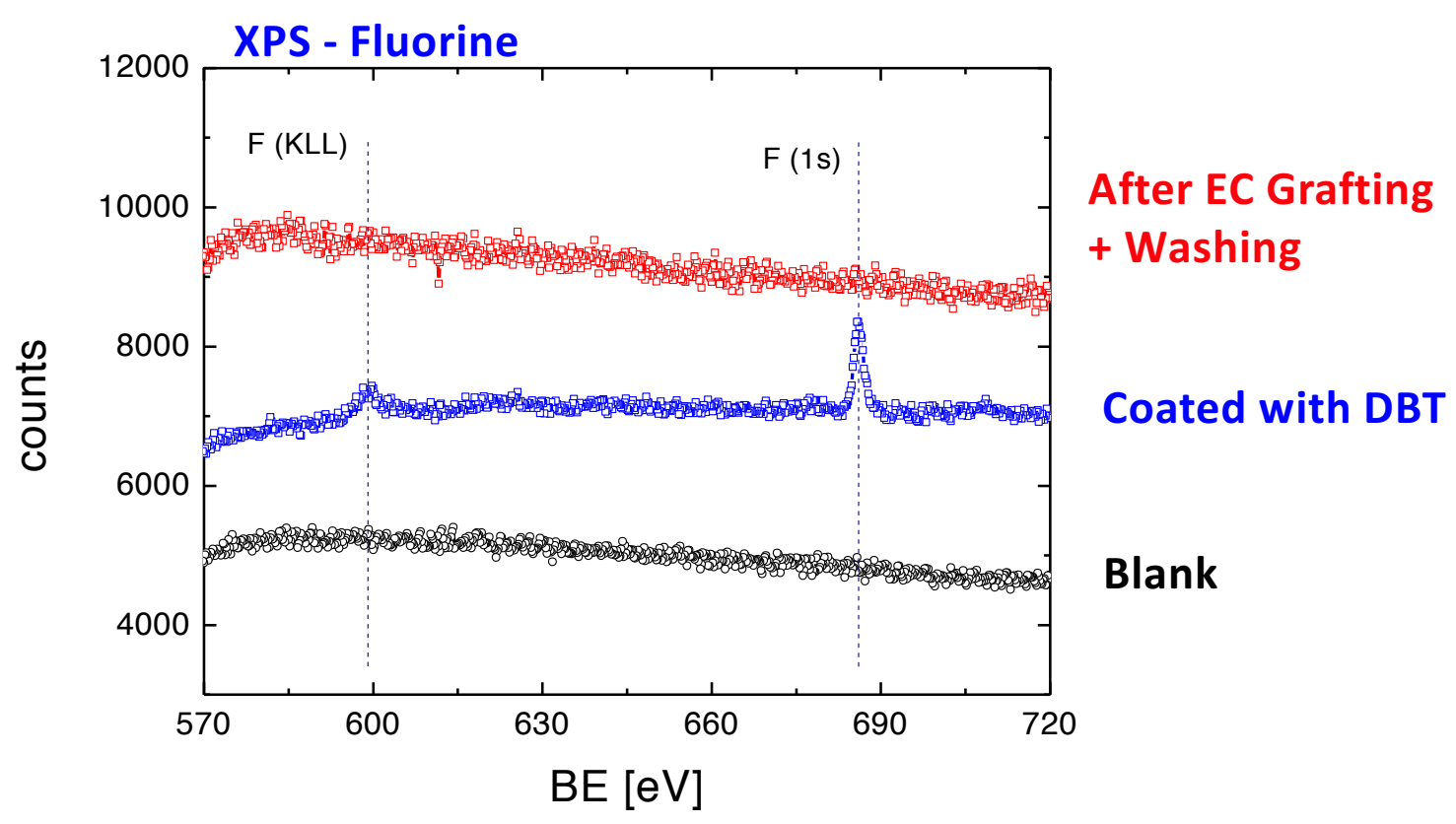

Figure 7. XPS spectra of graphene/SiOx substrate before and after spin coating of DBT, electrochemical grafting and washing. 